\title{
Two-proton femtoscopy at STAR
}

\author{
Hanna Paulina Zbroszczyk for the STAR Collaboration* \\ Warsaw University of Technology, Faculty of Physics, Koszykowa 75, 00662 Warsaw, Poland \\ E-mail: gos@if.pw.edu.pl
}

Two-particle femtoscopy allows one to study the properties of matter created in heavy ion collisions. It makes the study of space-time evolution of the source possible and may be applied to many different combinations of hadron pairs. Two-proton femtoscopy enables to extract the radii of produced sources which, compared to those obtained from pion studies, provide additional information about source characteristics. In this paper we present the correlation functions obtained for protons and antiprotons for $\mathrm{Au}+\mathrm{Au}$ collisions at $\sqrt{s_{N N}}=7.7,11.5,39,62.4$, and $200 \mathrm{GeV}$.

The Seventh Workshop on Particle Correlations and Femtoscopy

September 20 - 242011

University of Tokyo, Japan

\footnotetext{
*Speaker.
} 


\section{Introduction}

Solenoidal Tracker At RHIC (STAR) is one of experiments performed at Relativistic Heavy Ion Collider (RHIC) at Brookhaven National Laboratory (BNL). The main goal of the STAR experiment is to measure the properties of matter created in heavy ion collisions at ultrarelativistic energies, and to study the formation of matter and the properties of interactions between hadrons. For over ten years, STAR has been collecting data from $\mathrm{Au}+\mathrm{Au}$ collisions at different energies: 7.7, 11.5, 19.6, 39, 62.4, 130, and $200 \mathrm{GeV}$. The Beam Energy Scan (BES) program [1], which includes colliding energies below $\sqrt{s_{N N}}=62.4 \mathrm{GeV}$, is mainly focused on exploration of the phase transition between hadron gas and quark-gluon plasma, and on the search for the critical point of quantum chromodynamics.

Analyzing the momentum correlations, it is possible to access information about space-time characteristics of source which cannot be measured directly. Correlations of identical baryons as protonproton or antiproton-antiproton reflect the properties of the Quantum Statistics (QS) and of the Final State Interactions (FSI): Coulomb and strong. Correlations of proton-antiproton pairs are sensitive to the FSI only. The correlation effect is determined by the distance separating emission points in space and time. In this paper we present results for proton femtoscopy from $\mathrm{Au}+\mathrm{Au}$ collisions at $\sqrt{s_{N N}}=7.7,11.5,39,62.4$, and $200 \mathrm{GeV}$.

Due to high multiplicities of protons, two-proton correlations were intensively measured before at AGS (BNL), SPS (CERN) and SIS (GSI) energies [2]. The STAR measurement, in addition to exploring new collision energies, has the advantage of a simultaneous measurement of all three combinations of protons and antiprotons.

\section{Details of data analysis}

The correlations of two particles are measured in the momentum difference variable $k^{*}=$ $\frac{Q_{\text {inv }}}{2}=\frac{1}{2} \sqrt{\left(p_{1}-p_{2}\right)^{2}-\left(E_{1}-E_{2}\right)^{2}}$. The correlation function is defined as a ratio: $C\left(k^{*}\right)=\frac{A\left(k^{*}\right)}{B\left(k^{*}\right)}$. Pairs of correlated particles which come from the same event enter into the numerator $A\left(k^{*}\right)$ and the pairs of uncorrelated particles from different events into the denominator $B\left(k^{*}\right)$. All particles are registered by the main STAR detector, the Time Projection Chamber (TPC). All minimum bias events are divided according to the percentage of total cross-section of the collision into three centralities: central (0-10\%), midcentral (10-30\%), and peripheral (30-80\%). All particles are selected using the energy loss in the detector $(d E / d x)$. Protons and antiprotons are chosen in the momentum range: $0.4<p_{T}<0.8 \mathrm{GeV} / \mathrm{c}$ and in the rapidity window $|y|<0.5$. Each track is extrapolated to the primary vertex. If the shortest distance between track and the vertex exceeds $3 \mathrm{~cm}$, the track is discarded. This removes a significant fraction of non-primary track candidates. The effect of tracks splitting and merging is also taken into account, such tracks as well as these contributed to $e+e-$ candidates from $\gamma$ conversion are discarded. The background of the correlation function is constructed by combining particles from different events with similar Z-vertex position and multiplicity. All data are corrected for purity. In order to fit experimental correlation functions the CorrFit tool [3] is used. 


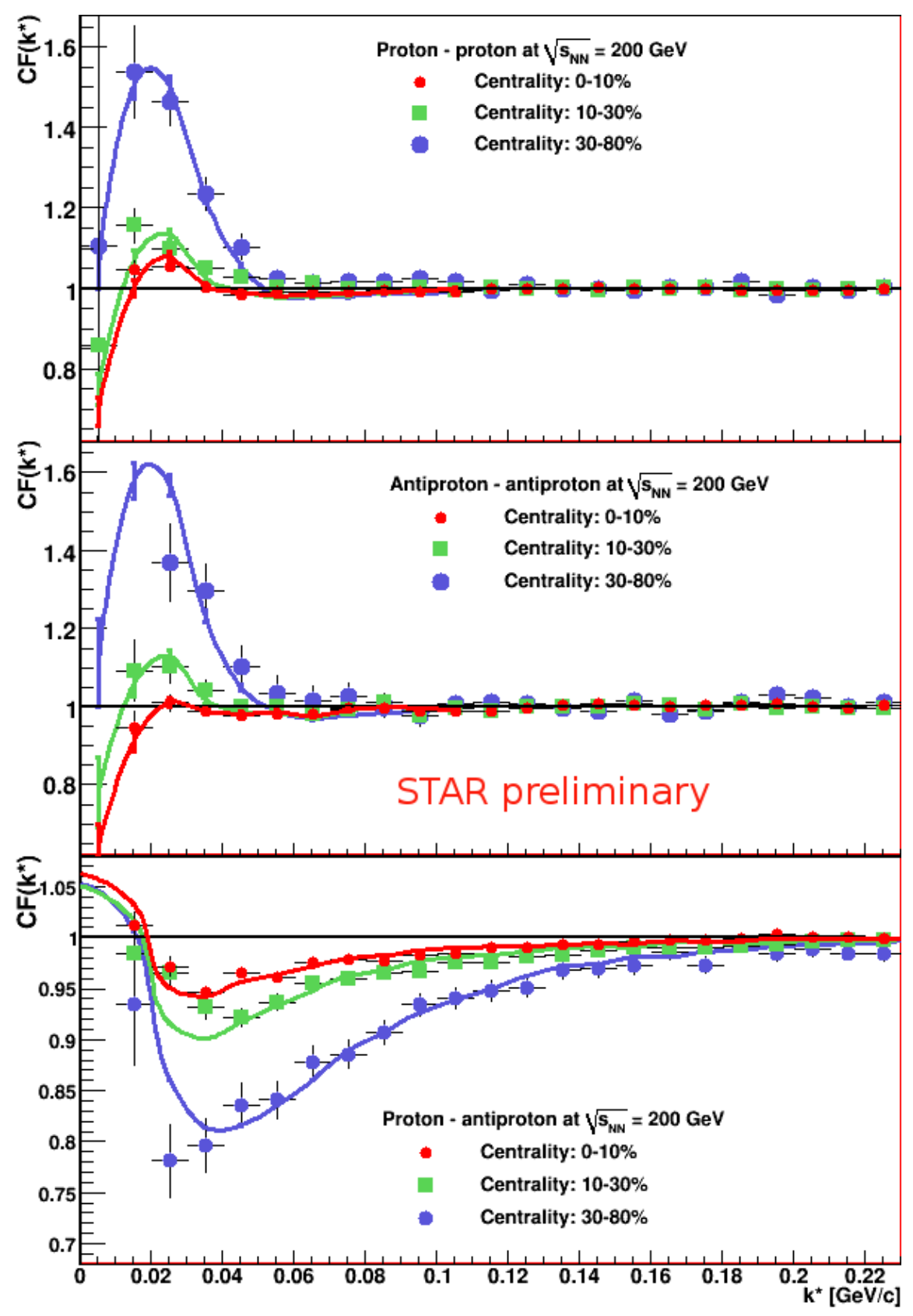

Figure 1: The correlation functions proton-proton (top), antiproton-antiproton (middle), and protonantiproton (bottom) at $\sqrt{s_{N N}}=200 \mathrm{GeV}$ for three centralities: $0-10 \%$ (red), $10-30 \%$ (green) and 30-80\% (blue) with theoretical fits (solid lines). 


\section{Results for $\mathrm{Au}+\mathrm{Au}$ collisions at $\sqrt{s_{N N}}=62.4$ and $200 \mathrm{GeV}$}

Figure 1 presents the correlation functions of proton-proton, antiproton-antiproton, and protonantiproton for three different centralities for collision energy $\sqrt{s_{N N}}=200 \mathrm{GeV}$. All results include necessary corrections: for purity, for detector resolution effects, and for residual correlations (RC). The latter correction accounts for the mutual correlations between hyperons which subsequently decay into protons. It is important because a significant fraction (around 50\%) of (anti)protons originate from such decays. For all systems the width and height of the correlation function decrease - reflecting the source size increase - with centrality. Each correlation function is fitted with a theoretical formula taking into account FSI and QS (for identical particle combinations) assuming a Gaussian source distribution, and the source size is extracted. Table 1 shows the source sizes extracted from proton-proton and proton-antiproton correlation functions for $\mathrm{Au}+\mathrm{Au}$ collision at $\sqrt{s_{N N}}=62.4 \mathrm{GeV}$. Antiproton-antiproton systems are not included due to limited statistics. Table 2 presents the analogous results for $\sqrt{s_{N N}}=200 \mathrm{GeV}$. The values of fit for all identical systems give consistent description within all centralities and energies. The agreement between results for identical and nonidentical particle correlations is good, however small discrepancies do remain. The stability of the fit is checked by varying the applied corrections by $\pm 10 \%$. The results indicate that all corrections should be applied together as all of them influence strongly the measured correlation functions, as described in details in [4].

Table 1: Source sizes extracted from proton-proton and proton-antiproton correlations in $\mathrm{Au}+\mathrm{Au}$ at $\sqrt{s_{N N}}=$ $62 \mathrm{GeV}$ at three centralities. The statistical errors, as well as the systematic errors from the purity, resolution and RC corrections, are quoted separately.

\begin{tabular}{|c|c|c|}
\hline Centrality & $p-p[\mathrm{fm}]$ & $p-\bar{p}[\mathrm{fm}]$ \\
\hline \hline $30-80 \%$ & $2.48_{-0.13-0.05-0.02-0.05}^{+0.13+0.06+0.02+0.05}$ & $2.33_{-0.13-0.05-0.02-0.05}^{+0.13+0.05+0.02+0.05}$ \\
\hline $10-30 \%$ & $3.23_{-0.12-0.05-0.02-0.06}^{+0.12+0.06+0.02+0.09}$ & $2.72_{-0.13-0.04-0.02-0.06}^{+0.13+0.05+0.02+0.04}$ \\
\hline $0-10 \%$ & $4.17_{-0.11-0.12-0.03-0.20}^{+0.11+0.11+0.03+0.09}$ & $3.53_{-0.12-0.03-0.01-0.05}^{+0.12+0.04+0.02+0.04}$ \\
\hline
\end{tabular}

Table 2: Source sizes extracted from proton-proton, antiproton-antiproton and proton-antiproton correlations in $\mathrm{Au}+\mathrm{Au}$ at $\sqrt{s_{N N}}=200 \mathrm{GeV}$ at three centralities. The statistical errors, as well as the systematic errors from the purity, resolution and RC corrections, are quoted separately.

\begin{tabular}{|c|c|c|c|}
\hline Centrality & $p-p[\mathrm{fm}]$ & $\bar{p}-\bar{p}[\mathrm{fm}]$ & $p-\bar{p}[\mathrm{fm}]$ \\
\hline $30-80 \%$ & $2.71_{-0.11-0.05-0.02-0.05}^{+0.11+0.05+0.02+0.06}$ & $2.59_{-0.13-0.07-0.05-0.13}^{+0.13+0.08+0.04+0.14}$ & $2.22_{-0.12-0.05-0.02-0.06}^{+0.12+0.05+0.02+0.05}$ \\
\hline $10-30 \%$ & $3.82_{-0.09-0.04-0.02-0.04}^{+0.09+0.05}$ & $4.02_{-0.09-0.07-0.04-0.12}^{+0.09+0.07+0.04+0.13}$ & $3.27_{-0.10-0.03-0.02-0.06}^{+0.10+0.08}$ \\
\hline $0-10 \%$ & $4.51_{-0.07-0.06-0.03-0.03+0.07}^{+0.07+0.07}$ & $5.05_{-0.08-0.06-0.04-0.11}^{+0.08+0.07+0.05+0.10}$ & $4.08_{-0.09-0.03+0.01+0.02+0.05}^{+0.09+0.03+0.00}$ \\
\hline
\end{tabular}

\section{Results for the BES program at $\sqrt{s_{N N}}=7.7,11.5$, and $39 \mathrm{GeV}$}

Figures 2 - 4 present proton-proton and proton-antiproton correlation functions for $\sqrt{s_{N N}}=$ 7.7, 11.5, and $39 \mathrm{GeV}$ for various collision centralities. Due to limited statistics the correlation functions of antiproton-antiproton pairs are not calculated. In order to extract source sizes, the best fitted, theoretical correlation functions are considered. Table 3 presents the results of the fits. 

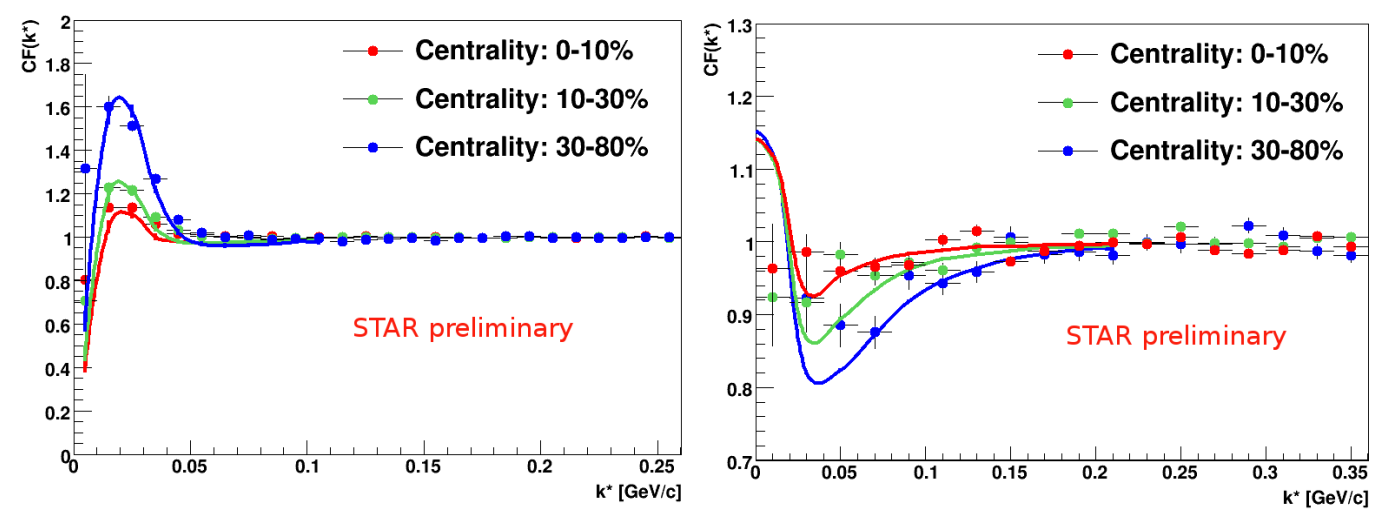

Figure 2: Proton-proton (left) and proton-antiproton (right) correlation functions, with the purity correction applied, for $\sqrt{s_{N N}}=7.7 \mathrm{GeV}$.
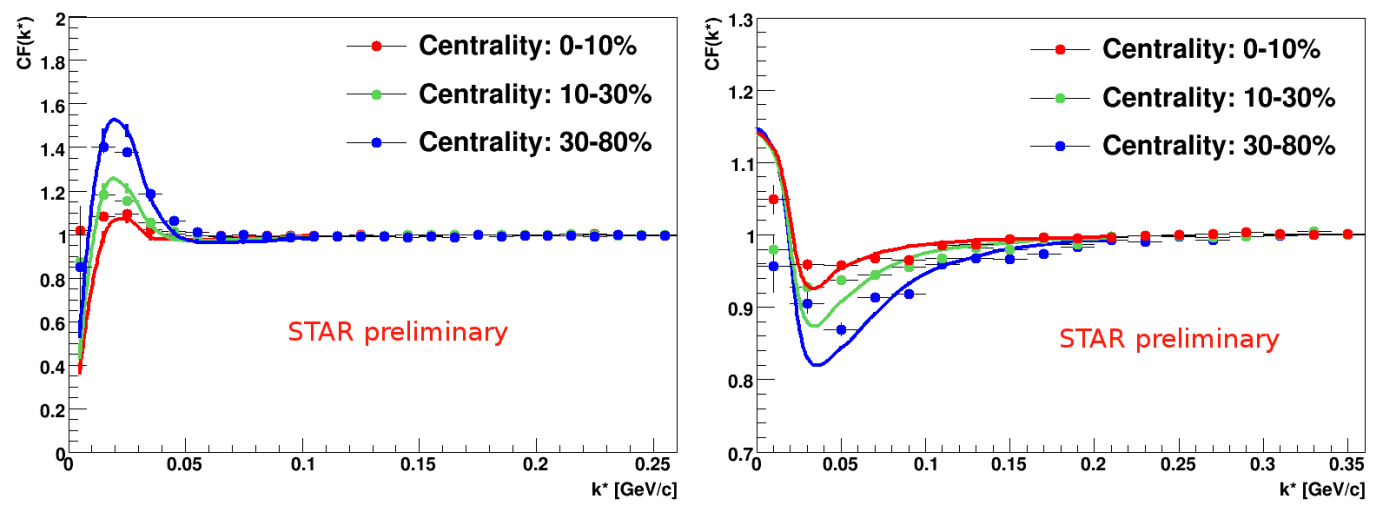

Figure 3: Proton-proton (left) and proton-antiproton (right) correlation functions, with the purity correction applied, for $\sqrt{s_{N N}}=11.5 \mathrm{GeV}$.
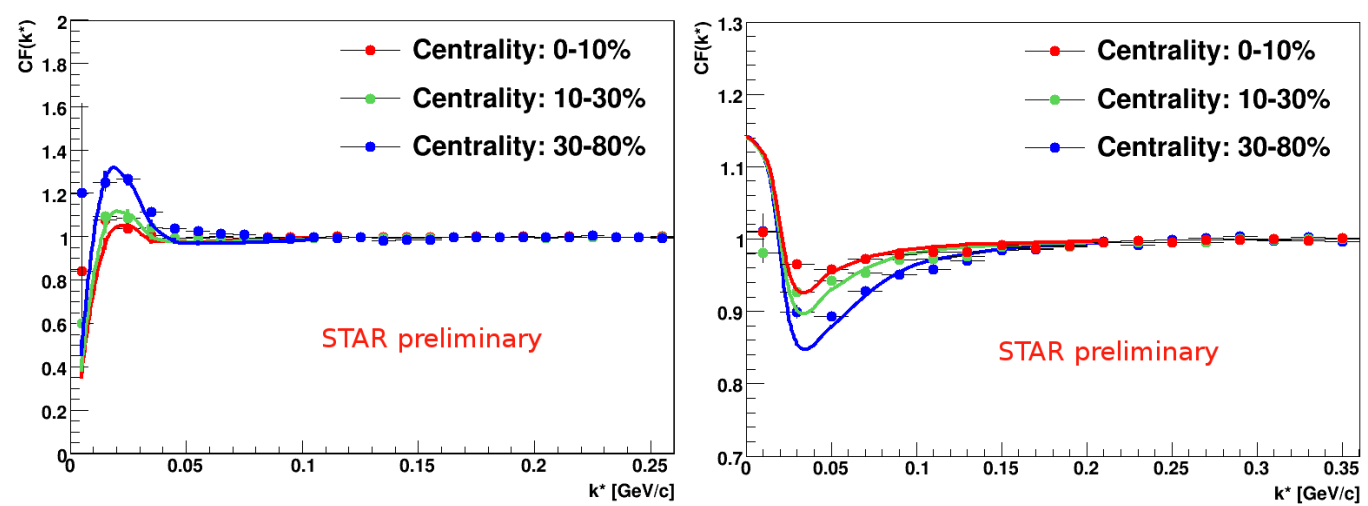

Figure 4: Proton-proton (left) and proton-antiproton (right) correlation functions, with the purity correction applied, for $\sqrt{s_{N N}}=39 \mathrm{GeV}$. 
For each collision energy and each centrality the extracted source sizes for proton-antiproton pairs are smaller than for identical proton combinations. These discrepancies originate from the fact that the RC correction has not yet been applied. For the RC correction, first the contribution of various decays of the proton yield must be determined. This can be done e.g. using UrQMD. The corresponding analysis is currently in progress.

Table 3: Source sizes estimated from proton-proton and proton-antiproton correlations at three energies. Only statistical errors are quoted.

\begin{tabular}{|c|c|c|}
\hline Energy / centrality & $p-p[\mathrm{fm}]$ & $p-\bar{p}[\mathrm{fm}]$ \\
\hline \hline 7.7 GeV: 0-10\% & $4.15 \pm 0.43$ & $3.98 \pm 0.23$ \\
\hline $10-30 \%$ & $3.62 \pm 0.21$ & $2.92 \pm 0.41$ \\
\hline $30-80 \%$ & $2.74 \pm 0.32$ & $2.21 \pm 0.43$ \\
\hline \hline $11.5 \mathrm{GeV}: 0-10 \%$ & $4.51 \pm 0.23$ & $3.98 \pm 0.13$ \\
\hline $10-30 \%$ & $3.62 \pm 0.11$ & $3.09 \pm 0.25$ \\
\hline $30-80 \%$ & $2.92 \pm 0.13$ & $2,39 \pm 0.34$ \\
\hline \hline 39 GeV: $0-10 \%$ & $4.68 \pm 0.16$ & $3.98 \pm 0.24$ \\
\hline $10-30 \%$ & $4.15 \pm 0.27$ & $3.45 \pm 0.36$ \\
\hline $30-80 \%$ & $3.45 \pm 0.34$ & $2.75 \pm 0.29$ \\
\hline \hline
\end{tabular}

\section{Summary}

We have presented the current status of the proton-proton, antiproton-antiproton, and protonantiproton femtoscopy for $\mathrm{Au}+\mathrm{Au}$ collisions at $\sqrt{s_{N N}}=200 \mathrm{GeV}$, and proton-proton, protonantiproton femtoscopy results for $\mathrm{Au}+\mathrm{Au}$ collisions at $\sqrt{s_{N N}}=7.7,11.5,39$, and $62.4 \mathrm{GeV}$ at STAR. Thanks to the large STAR dataset, all data could be divided into three centrality classes. For all studied energies, the source sizes extracted from proton-proton and antiproton-antiproton correlation functions agree with each other. The importance of the corrections for: purity, detector resolution effects, and residual correlations was demonstrated for data at collision energies $\sqrt{s_{N N}}$ $=62.4$ and $200 \mathrm{GeV}$. Not taking into consideration effects of RCs is reflected in discrepancies between the source sizes estimated for identical and nonidentical combinations of protons and antiprotons for collision energies $\sqrt{s_{N N}}=39 \mathrm{GeV}$ and lower.

\section{References}

[1] G. Odyniec, J. Phys. G37 (2010) 094028.

[2] J. Barrette et al., Phys. Rev. C60 (1999) 054905; H. Boggild et al., Phys. Lett. B458 (1999) 181-189; H. Appelshauser, Phys. Lett. B467(1999) 21-28; R. Kotte et al., Eur. J. Phys. A23 (2005) 271-278.

[3] A. Kisiel, Nukleonika: 2004 vol 49 suppl. 2 (2004) 81.

[4] H.P. Gos, Eur. Phys. J. C49 (2007) 75-80; H.P. Zbroszczyk, Phys. Part. Nucl. Lett. vol. 8, no. 9 (2011) 931-933. 